\title{
Hall algebras and quantum groups
}

\author{
Claus Michael Ringel \\ Fakultät für Mathematik, Universität Bielefeld, D-4800 Bielefeld 1, FRG
}

\section{Dedicated to Jacques Tits on his sixtieth birthday}

Let $R$ be a finite-dimensional representation-finite hereditary algebra over some field. Let $\Delta$ be its type, this is a disjoint union of Dynkin diagrams [DR]. Let $\Phi^{+}$be the set of positive roots for $\Delta$. Given $\alpha \in \Phi^{+}$, there is (up to isomorphism) a unique indecomposable $R$-module $M(\alpha)$ with dimension vector $\alpha$. Given a function $a: \Phi^{+} \rightarrow \mathbb{N}_{0}$, let $M(a)$ denote the direct sum of $a(\alpha)$ copies of the various $M(\alpha)$ with $\alpha \in \Phi^{+}$; in this way, the isomorphism classes of $R$-modules of finite length correspond bijectively to the functions $a: \Phi^{+} \rightarrow \mathbb{N}_{0}$. Given $a$, $b, c: \Phi^{+} \rightarrow \mathbb{N}_{0}$, we denote by $\varphi_{M(a), M(c)}^{M(b)}=\varphi_{a c}^{b}$ the corresponding Hall polynomial [R1], it is a polynomial with integer coefficients which counts (for finite $R$ ) the number of filtrations of $M(b)$ with factors $M(a)$ and $M(c)$. If $A$ is an arbitrary commutative ring, and $q \in A$, we define the Hall algebra $\mathscr{H}(R, A, q)$ as the free $A$-module with basis $\left(u_{[M]}\right)_{[M]}$ indexed by the isomorphism classes of $R$-modules of finite length, with multiplication

$$
u_{[N]} u_{\left[N^{\prime}\right]}=\sum_{[M]} \varphi_{N N^{\prime}}^{M}(q) u_{[M]}
$$

in this way, we obtain a (usually non-commutative) associative ring with 1 . In [R 2], we have shown that we may identify $\mathscr{H}(R, \mathbb{C}, 1)$ with the universal enveloping algebra $U\left(\mathbf{n}_{+}\right)$of $\mathbf{n}_{+}$, where $\mathbf{g}=\mathbf{n}_{-} \oplus \mathbf{h} \oplus \mathbf{n}_{+}$is a triangular decomposition of the semisimple complex Lie algebra of type $\Delta$.

It would be of interest to find a natural enlargement of $\mathscr{H}(R, \mathbb{C}, 1)$ in order to obtain $U(\mathbf{g})$ itself. As we will show in Sect. 3, there is a canonical way for obtaining at least $U\left(\mathbf{b}_{+}\right)$, where $\mathbf{b}_{+}=\mathbf{h} \oplus \mathbf{n}_{+}$is the Borel algebra. Let $S_{1}, \ldots, S_{s}$ be a complete set of simple $R$-modules. If $M$ is an $R$-module of finite length, let $(\operatorname{dim} M)_{i}$ be the Jordan-Hoelder multiplicity of $S_{i}$ in $M$. Then the map $\delta_{i}$ of $\mathscr{H}(R, A, q)$ into itself defined by $\delta_{i}\left(u_{[M]}\right)=(\operatorname{dim} M)_{i} u_{[M]}$ is a derivation, so we may define the skew polynomial ring

$$
\mathscr{H}^{\prime}(R, A, q)=\mathscr{H}(R, A, q)\left[T_{i}, \delta_{i}\right]_{i}
$$

in $s$ variables $T_{1}, \ldots, T_{s}$. Since $\mathscr{H}(R, \mathbb{C}, 1)$ is isomorphic to $U\left(\mathbf{n}_{+}\right)$, it follows that $\mathscr{H}^{\prime}(R, \mathbb{C}, 1)$ is isomorphic to $U\left(\mathbf{b}_{+}\right)$. 
Instead of dealing with the degenerate Hall algebra $\mathscr{H}^{\prime}(R, \mathbb{C}, 1)$, we are going to consider the generic Hall algebra $\mathscr{H}^{\prime}(R, \mathbb{C}[q], q)$, where $\mathbb{C}[q]$ is the polynomial ring in the indeterminate $q$, or its completion

$$
\widehat{\mathscr{H}}^{\prime}(R)=\varliminf_{m} \lim _{m} \mathscr{H}^{\prime}\left(R, \mathbb{C}[q] /(q-1)^{m}, q\right),
$$

this is an algebra over the power series ring $\mathbb{C}[[q-1]]$. Our aim is to give a complete description of $\widehat{\mathscr{H}^{\prime}}(R)$ by generators and relations.

In $\mathbb{C}[[q-1]]$, the element $\ln q=\sum_{m \geqq 1}(-1)^{m+1} \frac{1}{m}(q-1)^{m}$ is a multiple of $q-1$, thus, for $c \in \mathbb{C}$, the element $\exp (c \ln q)=\sum_{m \geqq 0} \frac{1}{m !} c^{m}(\ln q)^{m}$ is defined. We also will write $q^{c}$ instead of $\exp (c \ln q)$, in particular, both $q^{\frac{1}{2}}$ and $q^{-\frac{1}{2}}$ are defined. We denote by $\left[\begin{array}{l}n \\ t\end{array}\right]_{q}=\frac{\varphi_{n}}{\varphi_{t} \varphi_{n-t}}$ the Gauss polynomials, where $\varphi_{n}=(1-q) \ldots\left(1-q^{n}\right)$.

Let $\left(a_{i j}\right)_{i j}$ be the Cartan matrix of type $\Delta$, and $\left(f_{i}\right)_{i}$ the (minimal) symmetrization of $A$ (so that $f_{i} a_{i j}=f_{j} a_{j i}$ ). Let $q_{i}=q^{f_{i}}$. We will show that $\widehat{\mathscr{H}}^{\prime}(R)$ is, as a complete $\mathbb{C}[[q-1]]$-algebra, generated by elements $H_{1}, \ldots, H_{s}, X_{1}, \ldots, X_{s}$ subject to the relations

$$
\begin{aligned}
& {\left[H_{i}, H_{j}\right]=0 \text {, }} \\
& {\left[H_{i}, X_{j}\right]=a_{i j} X_{j} \text {, }} \\
& \sum_{i=0}^{n}(-1)^{t}\left[\begin{array}{l}
n \\
t
\end{array}\right]_{q_{i}} q_{i}^{-\frac{t(n-t)}{2}} \mathrm{X}_{\mathrm{i}}^{\mathrm{t}} \mathrm{X}_{\mathrm{j}} \mathrm{X}_{\mathrm{i}}^{\mathrm{n}-1}=0, \quad \text { with } n=1-a_{i j}, \quad \text { and } i \neq j .
\end{aligned}
$$

This description shows that $\widehat{\mathscr{H}}(R)$ is precisely the quantization $U_{h}\left(\mathbf{b}_{+}\right)$of $U\left(\mathbf{b}_{+}\right)$as described by Drinfeld in his Berkeley lecture [D] (with $h=\ln q$ ). In particular, it follows that $\widehat{\mathscr{H}}^{\prime}(R)$ is a Hopf algebra.

The Hall algebra approach yields a rather natural interpretation of the awkward relations above. Consider besides

$$
\rho_{n}(q, X, Y)=\sum_{t=0}^{n}(-1)^{t}\left[\begin{array}{l}
n \\
t
\end{array}\right]_{q} q^{-\frac{t(n-t)}{2}} X^{t} Y X^{n-t}
$$

also the polynomials

$$
\begin{aligned}
& \rho_{n}^{+}(q, X, Y)=\sum_{t=0}^{n}(-1)^{t}\left[\begin{array}{l}
n \\
t
\end{array}\right]_{q} q^{\left(\begin{array}{l}
t \\
2
\end{array}\right) X^{t} Y X^{n-t},} \\
& +\rho_{n}(q, X, Y)=\sum_{t=0}^{n}(-1)^{t}\left[\begin{array}{l}
n \\
t
\end{array}\right]_{q} q^{\left(\begin{array}{l}
t \\
2
\end{array}\right) X^{n-t} Y X^{t} .}
\end{aligned}
$$

Observe that $\mathscr{H}(R, \mathbb{C}[[q-1]], q)$ is a subring of $\widehat{\mathscr{H}}^{\prime}(R)$. The elements $X_{1}, \ldots, X_{s}$ of $\widehat{\mathscr{H}}^{\prime}(R)$ are suitable multiples of the canonical generators $u_{1}=u_{\left[s_{1}\right]}, \ldots, u_{s}=u_{\left[s_{s}\right]}$ of $\mathscr{H}(R, \mathbb{C}[[q-1]], q)$. The relations which are satisfied by 
$u_{1}, \ldots, u_{s}$ and which give rise to the relations above, depend on the orientation of $\Delta$ defined by $R$. So assume $\operatorname{Ext}^{1}\left(S_{i}, S_{j}\right)=0$ for some pair $i \neq j$. We will show that

$$
\rho_{1-a_{i j}}^{+}\left(q_{i}, u_{i}, u_{j}\right)=0, \quad \text { and }{ }^{+} \rho_{1-a_{j i}}\left(q_{j}, u_{j}, u_{i}\right)=0 \text {, }
$$

and a simple substitution transforms these relations into the symmetric ones involving $\rho$ instead of $\rho^{+}$and ${ }^{+} \rho$. The relations involving $\rho^{+}$and ${ }^{+} \rho$ will be shown in a quite general setting in Sect. 2 . In order to do so, we will introduce in Sect. 1 the composition algebra $\mathscr{C}(R)$ for an arbitrary ring $R$.

The reader should be aware that $q$ (and $q_{i}=q^{f_{i}}$ ) may denote an integer, or a variable, in different parts of the paper.

The author is endebted to R. Dipper, B. Pareigis, and L. Scott for helpful comments: they insisted that there should be a strong relationship between Hall algebras as presented in [R 1] and [R2] and the recent advances on Hopf algebras and quantum groups.

\section{Composition algebras}

Let $R$ be any ring, let $\mathscr{S}$ be the set of isomorphism classes of finite simple $R$-modules (where 'finite' means: having only a finite number of elements). Let $\mathscr{W}(R)$ be the free semigroup with basis $\mathscr{S}$, thus the elements of $\mathscr{W}(R)$ are words of the form $w=\left[S_{1}\right]\left[S_{2}\right] \ldots\left[S_{t}\right]$, where $S_{1}, \ldots, S_{t}$ are finite simple $R$-modules, and $\left[S_{i}\right]$ denotes the isomorphism class of $S_{i}$; here, $t$ is the length of the word $w$, and there is a unique word of length zero (denoted by 1 ). We denote by $\mathscr{A}(R)$ the free (associative) algebra with basis $\mathscr{S}$. Clearly, the additive group of $\mathscr{A}(R)$ is the free abelian group with basis $\mathscr{W}(R)$. Given an element $w \in \mathscr{W}(R)$, say $w=\left[S_{1}\right] \ldots\left[S_{t}\right]$, and an $R$-module $M$, let $\langle w \mid M\rangle$ denote the number of filtrations

$$
M=M_{0} \supset M_{1} \supset \ldots \supset M_{t}=0
$$

such that $M_{i-1} / M_{i} \cong S_{i}$. (The number of such filtrations always is finite: if $M$ has at least one such filtration, then $M$ is a finite module, and so has only finitely many submodules.) In general, given $\sum_{i=1}^{n} \lambda_{i} w_{i} \in \mathscr{A}(R)$, with $\lambda_{i} \in \mathbb{Z}$,
$w_{i} \in \mathscr{W}(R)$, and an $R$-module $M$, we define

$$
\left\langle\sum_{i=1}^{n} \lambda_{i} w_{i} \mid M\right\rangle=\sum_{i=1}^{n} \lambda_{i}\left\langle w_{i} \mid M\right\rangle
$$

Let $\mathscr{I}(R)$ be the set of all $a \in \mathscr{A}(R)$, with $\langle a \mid M\rangle=0$ for all $R$-modules $M$. This is an ideal of $\mathscr{A}(R)$. (For $a \in \mathscr{A}(R)$ and $S$ a finite simple $R$-module, $\langle[S] a \mid M\rangle$ $=\sum_{U}\langle a \mid M\rangle$, where the summation ranges over all submodules $U$ of $M$ such 
that $M / U \cong S$; similarly, $\langle a[S] \mid M\rangle=\sum_{V}\langle a \mid M / V\rangle$, where the summation ranges over all submodules $V$ of $M$ with $M / V$ isomorphic to $S$.) Define

$$
\mathscr{C}(R)=\mathscr{A}(R) / \mathscr{I}(R)
$$

the composition algebra of $R$. Note that $\langle-\mid-\rangle$ yields a bilinear form

$$
\mathscr{C}(R) \times K(R-\text { fin }) \rightarrow \mathbb{Z}
$$

Assume that the ring $R$ is finitary, so that the Hall algebra $\mathscr{H}(R)$ is defined. Consider the ring homomorphism $\eta: \mathscr{A}(R) \rightarrow \mathscr{H}(R)$ sending $[S]$ to $u_{[S]}$. Then $\mathscr{I}(R)=$ ker $\eta$. (For, $\quad \eta\left(\left[S_{1}\right] \ldots\left[S_{t}\right]\right)=\sum_{[M]} F_{S_{1}, \ldots, s_{t}}^{M} u_{[M]} \quad$ and $F_{S_{1}, \ldots, S_{t}}^{M}$ $=\left\langle\left[S_{1}\right] \ldots\left[S_{t}\right] \mid M\right\rangle$; therefore, given $a \in \mathscr{A}(R)$, we have $\eta(a)=\sum_{[M]}\langle a \mid M\rangle u_{[M]}$.) As a consequence, we can identify $\mathscr{C}(R)$ with the subring of $\mathscr{H}(R)$ generated by the elements of the form $u_{[\mathrm{S}]}$ with $[S] \in \mathscr{S}$.

\section{The fundamental relations}

Let $R$ be a finitary ring. Let $S_{i}(i \in I)$ be a complete set of finite simple $R$-modules (thus, they are pairwise non-isomorphic, and any finite simple $R$-module is isomorphic to one of them). We assume that $\operatorname{Ext}^{1}\left(S_{i}, S_{i}\right)=0$ for all $i$. Let $q_{i}$ $=\left|\operatorname{End}\left(S_{i}\right)\right|$. Let $i \neq j$ with $\operatorname{Ext}^{1}\left(S_{i}, S_{j}\right)=0$, and

thus $q_{i}^{a_{i j}}=q_{j}^{a_{i j}}$.

$$
\begin{aligned}
& a_{i j}=-\operatorname{dim} \operatorname{Ext}^{1}\left(S_{j}, S_{i}\right) \text { End }\left(S_{i}\right), \\
& a_{i j}^{\prime}=-\operatorname{dim}_{\operatorname{End}\left(S_{j}\right)} \operatorname{Ext}^{1}\left(S_{j}, S_{i}\right),
\end{aligned}
$$

Proposition. Both elements $\rho_{1-a_{i j}}^{+}\left(q_{i},\left[S_{i}\right],\left[S_{j}\right]\right)$ and ${ }^{+} \rho_{1-a_{i}^{i},}\left(q_{j},\left[S_{j}\right],\left[S_{i}\right]\right)$ belong to $\mathscr{I}(R)$.

Proof. We first consider $\rho^{+}$. We are going to calculate

$$
a_{t}(M):=\left\langle\left[S_{i}\right]^{t}\left[S_{j}\right]\left[S_{i}\right]^{n-t} \mid M\right\rangle
$$

for an arbitrary module $M$. We may assume that $M$ is of length $n+1$, with one composition factor $S_{j}$, the remaining ones of the form $S_{i}$. Since $\operatorname{Ext}^{1}\left(S_{i}, S_{i}\right)$ $=0=\operatorname{Ext}^{1}\left(S_{i}, S_{j}\right)$, we can decompose $M=N \oplus d S_{i}$, with $N$ indecomposable and some $0 \leqq d \leqq n$. The radical $N^{\prime}$ of $N$ is isomorphic to $(n-d) S_{i}$, and $N / N^{\prime} \cong S_{j}$. Since $\operatorname{dim} \operatorname{Ext}^{1}\left(S_{j}, S_{i}\right)_{\operatorname{End}\left(S_{i}\right)}=n-1$, it follows that $d \geqq 1$. Note that $M$ does not have a factor module isomorphic to $(d+1) S$, thus $a_{t}(M)=0$ for $t>d$. Therefore, we may assume $t \leqq d$. The composition series of $M$ we are interested in are of the form

$$
M=M_{0} \supset M_{1} \supset \ldots \supset M_{n+1}=0
$$



with $M_{t} / M_{t+1} \cong S_{j}$. In particular, $N \subseteq M_{t}$, since $M / N \cong d S_{i}$. There are $\frac{v_{d}}{v_{d-t}}\left(q_{i}\right)$
possibilities for choosing chains

$$
M=M_{0} \supset M_{1} \supset \ldots \supset M_{t} \supseteq N
$$

with $M_{i}$ maximal in $M_{i+1}$, for $1 \leqq i \leqq t$, where $v_{n}=v_{n}(T)=\frac{\varphi_{n}(T)}{(1-T)^{n}}$. Always, $M_{t}$ has a unique submodule $M_{t+1}$ with $M_{t} M_{t+1} \cong S_{j}$, and since $M_{t+1} \cong(n-t) S_{i}$, there are $v_{n-t}\left(q_{i}\right)$ composition series

$$
M_{t+1} \supset M_{t+2} \supset \ldots \supset M_{n} \supset M_{n+1}=0 .
$$

Thus

$$
a_{t}(M)=\frac{v_{d} v_{n-t}}{v_{d-t}}\left(q_{i}\right), \quad \text { for all } t \leqq d .
$$

We claim that for $1 \leqq d \leqq n$, we have

$$
\sum_{t=0}^{d}(-1)^{t}\left[\begin{array}{l}
n \\
t
\end{array}\right] T\left(\begin{array}{l}
t \\
2
\end{array}\right) \frac{v_{d} v_{n-t}}{v_{d-t}}=0
$$

But the evaluation of this polynomial at $q_{i}$ is just $\rho_{1-a_{i j}}^{+}\left(q_{i},\left[S_{i}\right],\left[S_{j}\right]\right)$, so this will finish the first part of the proof. We use

$$
\left[\begin{array}{l}
n \\
t
\end{array}\right] \frac{v_{d} v_{n-t}}{v_{d-t}}=\frac{\varphi_{n}}{\varphi_{t} \varphi_{n-t}} \cdot \frac{\varphi_{d} \varphi_{n-t}}{\varphi_{d-t}} \cdot \frac{1}{(1-T)^{n}}=v_{n}\left[\begin{array}{l}
d \\
t
\end{array}\right]
$$

in order to rewrite the left hand side $(*)$. We recall from [M] (I.2.Ex.3) that

$$
E_{d}(T, X):=\sum_{t=0}^{d}\left[\begin{array}{l}
n \\
t
\end{array}\right] T\left(\begin{array}{l}
t \\
2
\end{array}\right) X^{t}=\prod_{i=0}^{d-1}\left(1+T^{i} X\right)
$$

Since $d \geqq 1$, the right hand side shows that $E_{d}(T,-1)=0$, therefore

$$
\sum_{t=0}^{d}(-1)^{t}\left[\begin{array}{l}
n \\
t
\end{array}\right] T\left(\begin{array}{l}
(t) \\
2
\end{array}\right) \frac{v_{d} v_{n-t}}{v_{d-t}}=v_{n} \sum_{t=0}^{d}(-1)^{t}\left[\begin{array}{l}
d \\
t
\end{array}\right] T\left(\begin{array}{l}
t \\
2
\end{array}\right)=v_{n} E_{d}(T,-1)=0
$$

In order to deal with ${ }^{+} \rho$, we may use a corresponding calculation. Alternatively, we may argue as follows: Without loss of generality, we may assume that $S_{i}, S_{j}$ are the only simple $R$-modules, thus $R$ is a finite ring, and, in fact a $k$-algebra for some finite field $k$. We apply the previous considerations to 
the dual modules $S_{j}^{*}, S_{i}^{*}$, which we consider as $R^{\text {op }}$-modules. This is possible, since $\operatorname{Ext}_{R^{\text {op }}}^{1}\left(S_{j}^{*}, S_{i}^{*}\right)=0$. Given an $R$-module $M$, we have

$$
\left\langle\left[S_{j}^{*}\right]^{t}\left[S_{i}^{*}\right]\left[S_{j}^{*}\right]^{n-t} \mid M^{*}\right\rangle=\left\langle\left[S_{j}\right]^{n-t}\left[S_{i}\right]\left[S_{j}\right]^{t} \mid M\right\rangle,
$$

this finishes the proof.

As a consequence, we see that $\mathscr{C}(R)$ always may be considered as a factor algebra of $\mathscr{A}(R) / \mathscr{I}(R)$.

\section{Adjunction of $\mathrm{Hom}_{\mathbb{Z}}(\mathrm{K}(\mathrm{R}), \mathbb{Z})$}

Let $R$ be a finitary ring. The class of all finite $R$-modules will be denoted by $R$-fin 0 . Recall that a function $d: R-$ fin $_{0} \rightarrow \mathbb{Z}$ is said to be additive on exact sequences provided $d(X)-d(Y)+d(Z)=0$ for any exact sequence $0 \rightarrow X \rightarrow Y \rightarrow Z \rightarrow 0$ in $R-$ fin $_{0}$.

Lemma. Let $d: R-$ fin $_{0} \rightarrow \mathbb{Z}$ be additive on exact sequences. Define an additive function $\delta_{d}: \mathscr{H}(R) \rightarrow \mathscr{H}(R)$ by $\delta_{d}\left(u_{[M]}\right)=d(M) u_{[M]}$, for any finite $R$-module $M$. Then $\delta_{d}$ is a derivation.

Proof. Let $N, N^{\prime}$ be finite $R$-modules. Then

$$
\begin{aligned}
\delta_{d}\left(u_{[N]} u_{\left[N^{\prime}\right]}\right) & =\delta\left(\sum_{[M]} F_{N, N^{\prime}}^{M} u_{[M]}\right)=\sum_{[M]} F_{N, N^{\prime}}^{M} d(M) u_{[M]} \\
& =\sum_{[M]} F_{N, N^{\prime}}^{M}\left(d(N)+d\left(N^{\prime}\right)\right) u_{[M]} \\
& =d(N) u_{[N]} u_{\left[N^{\prime}\right]}+u_{[N]} d\left(N^{\prime}\right) u_{\left[N^{\prime}\right]} \\
& =\delta_{d}\left(u_{[N]}\right) u_{\left[N^{\prime}\right]}+u_{[N]} \delta_{d}\left(u_{\left[N^{\prime}\right]}\right)
\end{aligned}
$$

As in the previous section, let $S_{i}, i \in I$ be a complete set of finite simple $R$-modules. For $i \in I$, and $M \in R$-fin , let $_{i}(M)=(\operatorname{dim} M)_{i}$ be the Jordan-Hoelder multiplicity of $S_{i}$ in $M$. Then $d_{i}$ is additive on exact sequences (and $\left(d_{i}\right)_{i}$ is a basis of the free abelian group of all functions $R$-fin $n_{0} \rightarrow \mathbb{Z}$ which are additive on exact sequences). So we obtain a set of derivations $\delta_{i}=\delta_{d_{i}}$ of $\mathscr{H}(R)$.

Let $\mathscr{H}^{\prime}(R)$ be obtained from $\mathscr{H}(R)$ by forming the skew polynomial ring

$$
\mathscr{H}^{\prime}(R)=\mathscr{H}(R)\left[T_{i}, \delta_{i}\right]_{i}
$$

defined by the commutation rules

$$
\begin{aligned}
{\left[T_{i}, T_{j}\right] } & =0, \\
{\left[T_{i}, u_{[M]}\right] } & =\delta_{i}\left(u_{[M]}\right)=(\operatorname{dim} M)_{i} u[M]
\end{aligned}
$$

for all $i, j \in I$, and all $M \in R$-fin . $_{\text {. }}$

Assume now that $R$ is representation-directed, let $A$ be an arbitrary commutative ring, and $q \in A$. Given a function $d: R$-fin fin $_{0} \rightarrow \mathbb{Z}$ which is additive on exact sequences, we define $\delta_{d}: \mathscr{H}(R, A, q) \rightarrow \mathscr{H}(R, A, q)$ by $\delta_{d}\left(u_{[M]}\right)=d(M) u_{[M]}$, and 
again we see that $\delta_{d}$ is a derivation. In particular, we obtain the derivations $\delta_{i}$ with $\delta_{i}\left(u_{[M]}\right)=(\operatorname{dim} M)_{i} u_{[M]}$, and we define

$$
\mathscr{H}^{\prime}(R, A, q)=\mathscr{H}(R, A, q)\left[T_{i}, \delta_{i}\right]_{i}
$$

with the same commutation rules as above.

\section{Completion}

Let $k$ be a finite field, let $R$ be a finite-dimensional $k$-algebra with centre $k$ which is representation-finite and hereditary. Let $\Delta$ be its type, it is a Dynkin diagram (since $R$ is supposed to be connected). Let $S_{1}, \ldots, S_{s}$ be the simple $R$-modules, we assume that they are indexed in such a way that $\operatorname{Ext}^{1}\left(S_{i}, S_{j}\right)=0$ for $j<i$. We define $a_{i i}=2$, and, for $j<i$

$$
\begin{aligned}
& a_{i j}=-\operatorname{dim} \operatorname{Ext}^{1}\left(S_{j}, S_{i}\right)_{\operatorname{End}\left(S_{i}\right)}, \\
& a_{j i}=a_{i j}^{\prime}=-\operatorname{dim}_{\operatorname{End}\left(S_{j}\right)} \operatorname{Ext}^{1}\left(S_{j}, S_{i}\right) .
\end{aligned}
$$

Thus, $A=\left(a_{i j}\right)_{i j}$ is the Cartan matrix of type $A$. Let $f_{i}=\operatorname{dim}_{k} \operatorname{End}\left(S_{i}\right)$, thus $\left(f_{i}\right)_{i}$ is the minimal symmetrization of $A$.

Let $\mathbb{C}[q]$ be the polynomial ring in the indeterminate $q$. We consider

$$
\hat{\mathscr{H}}(R)=\frac{\lim }{m} \mathscr{H}\left(R, \mathbb{C}[q] /(q-1)^{m}, q\right),
$$

and the corresponding ring $\widehat{\mathscr{H}}^{\prime}(R)$, both are algebras over the power series ring $\mathbb{C}[[q-1]]$. We are going to describe both algebras $\hat{\mathscr{H}}(R)$ and $\widehat{\mathscr{H}}^{\prime}(R)$ by generators and relations. Let $u_{i}=u_{\left[s_{i}\right]}$ and $q_{i}=q^{f_{i}}$, for $1 \leqq i \leqq s$.

Theorem. As a complete $\mathbb{C}[[q-1]]$-algebra, $\hat{\mathscr{H}}(R)$ is generated by $u_{1}, \ldots, u_{s}$, with relations $\rho_{1-a_{i j}}^{+}\left(q_{i}, u_{i}, u_{j}\right)=0={ }^{+} \rho_{1-a_{j i}}\left(q_{j}, u_{j}, u_{i}\right)$ for all $j<i$.

Proof. Let $\mathscr{A}(R, \mathbb{C}[q])=\mathscr{A}(R) \otimes_{\mathbb{Z}} \mathbb{C}[q]$, the free $\mathbb{C}[q]$-algebra with generators $\left[S_{1}\right], \ldots,\left[S_{s}\right]$, and consider the algebra homomorphism

$$
\eta: \mathscr{A}(R, \mathbb{C}[q]) \rightarrow \mathscr{H}=\mathscr{H}(R, \mathbb{C}[q], q)
$$

defined by $\eta\left(\left[S_{i}\right]\right)=u_{i}$. Let $\mathscr{J}$ be the ideal of $\mathscr{A}(R, \mathbb{C}[q])$ generated by the elements $\rho_{1-a_{i j}}^{+}\left(q_{i},\left[S_{i}\right],\left[S_{j}\right]\right)$, and ${ }^{+} \rho_{1-a_{j},}\left(q_{j},\left[S_{j}\right],\left[S_{i}\right]\right)$ for all $j<i$. According to Sect. 1 , we see that $\mathscr{J}$ belongs to the kernel of $\eta$, thus we obtain an algebra homomorphism

We denote by

$$
\bar{\eta}: \overline{\mathscr{A}}=\mathscr{A}(R, \mathbb{C}[q]) / \mathscr{J} \rightarrow \mathscr{H} .
$$

$$
\bar{\eta}_{m}: \overline{\mathscr{A}} /(q-1)^{m} \overline{\mathscr{A}} \rightarrow \mathscr{H} /(q-1)^{m} \mathscr{H}
$$

the induced map modulo $(q-1)^{m}$. According to [R 2], the map $\vec{\eta}_{1}$ is bijective. We consider $\bar{\eta}_{m}$ as a map of $A_{m}$-modules, where $A_{m}=\mathbb{C}[q] /(q-1)^{m}$. Now, 
$\mathscr{H} /(q-1)^{m} \mathscr{H}$ is a free $A_{m}$-module, thus with $\bar{\eta}_{1}$ also $\bar{\eta}_{m}$ is bijective. It follows that $\bar{\eta}$ induces an isomorphism

$$
\varliminf_{m} \overline{\mathscr{A}} /(p-1)^{m} \overline{\mathscr{A}} \rightarrow \varliminf_{m} \mathscr{\mathscr { H }} /(q-1)^{m} \mathscr{H}=\hat{\mathscr{H}}(R) .
$$

Corolary. As a complete $\mathbb{C}[[q-1]]$-algebra, $\widehat{\mathscr{H}}^{\prime}(R)$ is generated by the elements $T_{1}, \ldots, T_{s}, u_{1}, \ldots, u_{s}$ subject to the relations

$$
\left[T_{i}, T_{j}\right]=0, \quad\left[T_{i}, u_{j}\right]=\delta_{i j} u_{j}, \quad \text { for all } i, j,
$$

and

$$
\rho_{1-a_{i j}}^{+}\left(q_{i}, u_{i}, u_{j}\right)=0={ }^{+} \rho_{1-a_{j i}}\left(q_{j}, u_{j}, u_{i}\right), \quad \text { for all } j<i .
$$

Here, $\delta_{i j}$ is the Kronecker delta: $\delta_{i i}=1, \delta_{i j}=0$, for $i \neq j$.

\section{Revision of the relations}

We keep the assumptions of the last section. We want to change the generators of $\widehat{\mathscr{H}}^{\prime}(R)$ in order to obtain more familiar relations. First of all, let

$$
H_{i}:=\sum_{j=1}^{s} a_{i j} T_{j}
$$

Since the Cartan matrix $A=\left(a_{i j}\right)_{i j}$ is invertible, the $\mathbb{C}$-space of $\widehat{\mathscr{H}^{\prime}}(R)$ generated by $H_{1}, \ldots, H_{s}$ is the same as that generated by $T_{1}, \ldots, T_{s}$. Also, $\left[T_{i}, T_{j}\right]=0$ for all $i, j$ is equivalent to requiring $\left[H_{i}, H_{j}\right]=0$ for all $i, j$. Similarly, $\left[T_{i}, u_{j}\right]=\delta_{i j} u_{j}$ for all $i, j$ is equivalent to requiring $\left[H_{i}, u_{j}\right]=a_{i j} u_{j}$ for all $i, j$.

In order to rewrite the relations $\rho^{+}$and ${ }^{+} \rho$, we will replace the elements $u_{i}$ by suitable multiples $c_{i} u_{i}$, with $c_{i}$ invertible in $\widehat{\mathscr{H}}^{\prime}(R)$. Given an element $b \in \widehat{\mathscr{H}}^{\prime}(R)$, the element $\exp (b \ln q)=\sum_{m \geqq 0}(-1)^{m} \frac{1}{m !} b^{m}(\ln q)^{m} \in \widehat{\mathscr{H}}(R)$ is defined, since $\ln q$ is a multiple of $q-1$. If $b_{1}, b_{2} \in \widehat{\mathscr{H}}^{\prime}(R)$ commute, then $\exp \left(\left(b_{1}+b_{2}\right)\right.$ $\ln q)=\exp \left(b_{1} \ln q\right) \exp \left(b_{2} \ln q\right)$; in particular, any $\exp (b \ln q)$ is invertible in $\widehat{\mathscr{H}}^{\prime}(R)$, with inverse $\exp (-b \ln q)$.

For $1 \leqq i \leqq s$, let

$$
X_{i}:=\exp \left(-\frac{1}{2} \sum_{j=1}^{i-1} f_{i} a_{i j} T_{j} \ln q\right) u_{i}
$$

Theorem. As a complete $\mathbb{C}[[q-1]]$-algebra, $\widehat{\mathscr{H}^{\prime}}(R)$ is generated by the elements $H_{1}, \ldots, H_{s}, X_{1}, \ldots, X_{s}$, subject to the relations

$$
\begin{aligned}
{\left[H_{i}, H_{j}\right] } & =0, \\
{\left[H_{i}, X_{j}\right] } & =a_{i j} X_{j}, \\
\rho_{1-a_{i j}}\left(q_{i}, X_{i}, X_{j}\right) & =0, \quad \text { for } i \neq j .
\end{aligned}
$$


Proof. For $1 \leqq j<i \leqq s$, let $c_{i j}=\exp \left(-\frac{1}{2} f_{i} a_{i j} T_{j} \ln q\right)$, and $c_{i}=c_{i 1} \ldots c_{i, j-1}$ (with $c_{1}=1$ ), thus $X_{i}=c_{i} u_{i}$. For $j \neq s$, we have $c_{i j} u_{s}=u_{s} c_{i j}$, since $\left[T_{j}, u_{s}\right]=0$. On the other hand, $T_{i} u_{i}=u_{i} T_{i}+u_{i}=u_{i}\left(T_{i}+1\right)$ implies by induction that $T_{i}^{m} u_{i}=u_{i}\left(T_{i}+1\right)^{m}$ for all $m \geqq 1$. Therefore, for $c \in \mathbb{C}$

$$
\begin{aligned}
\exp \left(c T_{i} \ln q\right) u_{i} & =\sum_{m \geqq 0} \frac{1}{m !}\left(c T_{i} \ln q\right)^{m} u_{i}=\sum_{m \geqq 0} \frac{1}{m !} c^{m}(\ln q)^{m} u_{i}\left(T_{i}+1\right)^{m} \\
& =u_{i} \exp \left(c\left(T_{i}+1\right) \ln q\right)=u_{i} \exp \left(c T_{i} \ln q\right) \exp (c \ln q) \\
& =q^{c} \cdot u_{i} \exp \left(c T_{i} \ln q\right),
\end{aligned}
$$

thus we see that

$$
c_{i j} u_{j}=q_{i}^{-\frac{1}{2} a_{i j}} u_{j} c_{i j}
$$

For $j<i$, it follows that

$$
c_{i} u_{i}=u_{i} c_{i}, \quad c_{i} u_{j}=q_{i}^{-\frac{1}{2} a_{i j}} u_{j} c_{i}, \quad c_{j} u_{i}=u_{i} c_{j}, \quad c_{j} u_{j}=u_{j} c_{j},
$$

and therefore, for all $0 \leqq t \leqq n$,

$$
\begin{aligned}
& q_{i}^{\frac{1}{2} a_{z j}{ }^{t}} X_{i}^{t} X_{j} X_{i}^{n-t}=u_{i}^{t} u_{j} u_{i}^{n-t} c_{j} c_{i}^{n},
\end{aligned}
$$

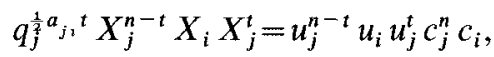

where we have used that $f_{i} a_{i j}=f_{j} a_{j i}$, thus $q_{i}^{a_{i j}}=q_{j}^{a_{j i}}$. We assume now that $n=$ $1-a_{i j}$. Then $\left(\begin{array}{l}t \\ 2\end{array}\right)+\frac{a_{i j} t}{2}=\frac{t(t-1)}{2}+\frac{(1-n) t}{2}=-\frac{t(n-t)}{2}$, and therefore

$$
\begin{aligned}
& \rho_{1-a_{i j}}\left(q_{i}, X_{i}, X_{j}\right)=\rho_{1-a_{i j}}^{+}\left(q_{i}, u_{i}, u_{j}\right) c_{j} c_{i}^{n}, \\
& \rho_{1-a_{j}}\left(q_{j}, X_{j}, X_{i}\right)={ }^{+} \rho_{1-a_{j}}\left(q_{j}, u_{j}, u_{i}\right) c_{j}^{n} c_{i} .
\end{aligned}
$$

This finishes the proof.

\section{References}

[D] Drinfeld, V.G.: Quantum groups. In: Proc. Int. Congr. Math. Berkeley 1986. Am. Math. Soc., 1987, pp. 798-820

[DR] Dlab, V., Ringel, C.M.: On algebras of finite representation type. J. Algebra 33, 306-394 (1975)

[M] Macdonald, I.G.: Symmetric functions and Hall polynomials. Clarendon Press: Oxford, 1979

[R1] Ringel, C.M.: Hall algebras. In: Topics in Algebra. Banach Centre Publ. 26. Warszawa (To appear)

[R 2] Ringel, C.M.: Hall polynomials for the representation-finite hereditary algebras. Adv. Math. (To appear) 